\title{
La situación de la tuberculosis en Chile y los actuales desafíos. Visita de la OPS al programa de control de la tuberculosis de Chile
}

\author{
TANIA HERRERA M.*
}

\section{Tuberculosis control and elimination program in Chile: present state and challenges}

In December 2012, the Tuberculosis Control and Elimination Program (Programa de Control $y$ Eliminación de la Tuberculosis-PROCET) of Chile, received an external Monitoring and Evaluation Mission of the Pan American Health Organization-PAHO (WHO Regional Office). The members of the Mission, together with national officials of PROCET, visited six Health Centers and presented their findings and recommendations to ministerial authorities. Even though the strengths that have led Chile to become a country with a low prevalence of tuberculosis (TB) were highlighted, they also pointed out the challenges that the program is facing, mainly the loss of priority that has suffered in recent years, which has resulted in a lack of resources and the weakening of operational activities. This has caused a dramatic slowing in the decrease of the incidence of TB in the last years. To face these challenges is the task that the Mission has given us and that PROCET endorses, with the aim of achieving the elimination of TB as a public health problem in Chile within the targets established by the Ministy of Health.

Key words: Tuberculosis control; Tuberculosis in Chile; PAHO evaluation.

\section{Resumen}

En diciembre de 2012, el Programa de Control y Eliminación de la Tuberculosis (PROCET) de Chile recibió una Misión de Monitoreo y Evaluación Externa de los Encargados de la Oficina Regional de Tuberculosis de la OPS. Los miembros de la Misión, en conjunto con los encargados nacionales del PROCET, visitaron seis Servicios de Salud y expusieron sus observaciones y recomendaciones a las autoridades ministeriales. Si bien destacaron las fortalezas que han llevado a Chile a convertirse en un país de baja prevalencia de tuberculosis, también advirtieron de los desafios que el Programa presenta en la actualidad, destacando especialmente la pérdida de prioridad que ha sufrido en los últimos años y que ha determinado tanto una falta de recursos, como el debilitamiento de las actividades operacionales. Esto se ha manifestado por un dramático enlentecimiento en la disminución de las tasas de incidencia de la TB en los últimos años. Enfrentar estos desafíos es la tarea que nos ha encomendado la Misión de la OPS, y que el PROCET hace suya, con el objetivo de poder alcanzar la eliminación de la Tuberculosis como problema de Salud Pública en Chile dentro de los plazos fijados por el mismo Ministerio de Salud.

Palabras clave: Control de la Tuberculosis; Tuberculosis en Chile; Evaluación OPS.

\section{Introducción}

En diciembre del año 2012, el Programa de Control y Eliminación de la Tuberculosis (PROCET) de Chile recibió una Misión de Monitoreo y Evaluación Externa de los Encargados de la Oficina Regional de Tuberculosis de la Organi- zación Panamericana de la Salud (OPS), cuyo objetivo fue conocer la Estrategia Nacional de Salud para Tuberculosis de Chile y evaluar la implementación de sus planes operativos.

Si bien Chile constituye un país de baja prevalencia de tuberculosis (TB), gracias a que en décadas anteriores experimentó una importante re-

* Médico, Directora del Programa de Control y Eliminación de la Tuberculosis- PROCET. Ministerio de Salud. 
ducción de las tasas de mortalidad y morbilidad, que le permitió traspasar el umbral de la etapa de eliminación el año 2000 (tasa de incidencia menor a 20 por 100.000 habitantes), aún se reportan alrededor de 2.500 enfermos por año, de los cuales casi 300 mueren a causa de esta enfermedad ${ }^{1}$. Además, durante la década pasada se ha producido un enlentecimiento de la curva de descenso de la incidencia de TB que no permitió alcanzar la meta propuesta en los Objetivos Sanitarios para la Década 2000-2010, que correspondía a la etapa de eliminación avanzada de la TB (tasa de incidencia menor a 10 por 100.000 habitantes) llegando sólo a 13,2 el año $2010^{1}$.

Así, en tanto que entre 1996 y el 2000 la tasa de incidencia de la tuberculosis disminuyó en promedio $7,5 \%$ por año, en el quinquenio 2000 2005 esta reducción fue de 4,2\% anualmente y entre 2005 y 2010 la reducción fue de sólo $1,3 \%$ anual en promedio ${ }^{1}$. Con la velocidad de reducción actual, la incidencia de la TB estará muy lejos de alcanzar la etapa de eliminación de la enfermedad como problema de Salud Pública, es decir de alcanzar una tasa menor a 5 casos por 100.000 habitantes, como corresponde a la meta planteada para el año 2020 por el Ministerio de Salud.

La misión de la OPS estuvo encabezada por el Dr. Rodolfo Rodríguez, Asesor Regional de Tuberculosis de OPS y contó con la presencia de expertos en distintos ámbitos del control de esta enfermedad: Dr. Jaime Bayona, experto en TB Multi-Drogo-Resistente (TB-MDR), Dra. Anna Volz, punto focal de TB-MDR en Latinoamérica, Dr. Ernesto Montoro, experto de Laboratorios de Koch, y Dr. Jorge Rodríguez, Jefe del Programa Nacional de Tuberculosis de Uruguay.

Los objetivos específicos que traía la misión eran los de evaluar los siguientes puntos:

- La implementación de la estrategia "Alto a la tuberculosis" y otras iniciativas recomendadas para los países de baja prevalencia.

- Las actividades de control de la TB en poblaciones vulnerables, como habitantes de zonas marginales, privados de libertad, pueblos originarios, etc.

- La aplicación de actividades de colaboración TB/VIH.

- El manejo clínico y programático de la TBMDR y su expansión nacional.

- La red de Laboratorios de TB y su respuesta a la situación epidemiológica del país.

La metodología del trabajo consistió en la creación de tres grupos en conjunto con los encargados nacionales del PROCET: un primer grupo visitó los Servicios de Salud (S.S.) de Arica e Iquique, un segundo grupo los S.S. de Reloncaví y Osorno, y un tercer grupo estuvo a cargo de la visita de los S.S. Metropolitano Central y Metropolitano Sur. Los S.S. visitados fueron acordados con los encargados nacionales y se eligieron en base a sus mayores tasas de incidencia (Arica, Iquique y Osorno) o al mayor número de pacientes afectados (Metropolitano Central y Sur). El S.S. de Reloncaví se visitó por su cercanía con Osorno.

Las visitas a los S.S. consistieron en reuniones con las autoridades y equipos técnicos de tuberculosis locales, donde se presentó la situación epidemiológica y operacional del programa a esos niveles, y en visitas a los establecimientos de la red asistencial -Hospitales, Centros de atención secundaria y Establecimientos de Atención Primaria- y a la Red de Laboratorios de TB.

Por otra parte, también se realizaron reuniones con las SEREMIs de Salud Metropolitana, Región de Los Lagos, Región de Arica y Parinacota y Región de Tarapacá, con Gendarmería en la Región Metropolitana, con el Programa de Prevención y Control del VIH/SIDA e ITS (Infecciones de transmisión sexual) en el Ministerio de Salud y con las autoridades del Instituto de Salud Pública (ISP).

Los miembros de la Misión presentaron al Subsecretario de Salud Pública sus principales observaciones y recomendaciones con respecto al funcionamiento del PROCET en Chile, las cuales se presentan a continuación ${ }^{2}$ :

\section{Fortalezas}

- La existencia de un Plan Nacional de Salud 2011-2020 en el cual se especifica como meta alcanzar la eliminación de la TB como Problema de Salud Pública el año 2020.

- La estructura de los servicios sanitarios que permite dar cobertura a las acciones del Programa de Control de la Tuberculosis.

- Los Equipos de TB, que en su mayoría están muy motivados y capacitados en los niveles intermedios y locales.

- Una Red de Laboratorios organizada, en la gran mayoría de forma descentralizada, dando cobertura a los niveles intermedio y local.

- Un sistema de registro e información que da respuesta a las necesidades del Programa en los diferentes niveles de salud mencionados.

- Conocimiento de la situación epidemiológica y operacional de la TB en los niveles mencionados.

- Conocimiento de los principales grupos vulnerables para TB en los Servicios de Salud visitados. 
- El tratamiento supervisado de los enfermos se realiza de forma total en todas las unidades visitadas (gran fortaleza).

- Conocimiento de la importancia del estudio de contactos y del estudio del VIH de los enfermos de TB.

- Existencia de una provisión adecuada de medicamentos para el tratamiento de los enfermos de TB, tanto de primera como de segunda línea, controlado para uso exclusivo de los mismos.

- Mantención en niveles bajos de la TB-MDR y con buen control en los Servicios visitados.

\section{Principales desafios}

Se observa que la TB ha perdido prioridad como problema de salud en Chile, fundamentalmente en los niveles intermedios, determinando una disminución de los recursos financieros y humanos. Esto ha traído como consecuencia la afectación de las principales actividades de control de la enfermedad. Es así como:

- Los equipos de tuberculosis de los Servicios de Salud están en su mayoría incompletos o con poco tiempo asignado al control de la TB. Esto ha generado que las principales actividades desarrolladas a este nivel, y que son fundamentales para el control de la tuberculosis, esto es: la supervisión y asesoría al nivel local y la capacitación del personal de salud, no se están realizando a cabalidad.

- Existe un importante deterioro de las actividades operacionales: disminución de la pesquisa de Sintomáticos Respiratorios, con cifras muy por debajo de las metas establecidas*; diagnóstico efectuado en una mayor proporción por los hospitales o servicios de urgencia en desmedro de la Atención Primaria; bajas tasas de curación con altas tasas de abandono y fallecidos**, y análisis de cohortes con alto número de casos excluidos y por ende no analizados.

- Bajo número de pacientes en los que se realiza la prueba de $\mathrm{VIH}^{* * *}$ y falta de registro de la quimioprofilaxis de los enfermos VIH positivos. Esta situación constituye un problema que incluye tanto al Programa Nacional de VIH como al PROCET.

- Existencia de un número importante de contactos de pacientes bacilíferos, e incluso con
TB-MDR, en los cuales no se logra realizar y registrar el estudio completo de los mismos.

- Fallas en la coordinación y apoyo regional a los centros penitenciarios ubicados en el área de atención de los Servicios de Salud.

- Deficiencias en la infraestructura de los laboratorios y dificultades en la adquisición de insumos y equipamiento, especialmente para aquellos laboratorios que centralizan las actividades de $\mathrm{TB}$, los que se encuentran al límite de su capacidad, aún con las bajas cifras de pesquisa actuales.

Cabe mencionar que, a pesar del deterioro en las actividades de localización de casos, existe un aumento discreto de casos en algunos de los Servicios visitados, lo que pudiera interpretarse como expresión de un aumento de la trasmisión por diagnóstico tardío de casos bacilíferos.

\section{Recomendaciones}

Es fundamental rescatar la prioridad de la gestión del PROCET en los 3 niveles de la organización y fundamentalmente en el nivel intermedio, lo que implica destinar los recursos financieros necesarios para el desarrollo de un plan de acción con vistas a rescatar la prioridad del Programa y poder alcanzar las metas del Plan 2011-2020 para la eliminación de la TB como Problema de Salud Pública. A partir del primer semestre de 2013, se recomienda establecer metas e indicadores para evaluar la marcha de este plan, comprometiendo a las autoridades de los Servicios de Salud con estos resultados.

Se requiere completar los equipos de TB en los Servicios de Salud y asignar las horas necesarias para el desarrollo de las actividades de control. Estos equipos deben realizar las labores de capacitación y supervisión a los niveles locales para incrementar la pesquisa de sintomáticos respiratorios con calidad según las metas establecidas, al mismo tiempo que se mejoran las tasas de curación (con disminución de abandonos y fallecidos) y el estudio de los contactos. De esta manera se pretende rescatar la tendencia de la morbi-mortalidad por TB, alcanzando los porcentajes de disminución de años precedentes a 2007.

Es necesario establecer la coordinación con el Programa de VIH, logrando bilateralmente mejor información sobre la situación de la co-infección

\footnotetext{
* La meta para el índice de pesquisa es de 50 baciloscopías por 1000 consultas de morbilidad adulto. El Índice de pesquisa el año 2010 fue de 14,9.

** Menor a $85 \%$ para los últimos 5 años. La meta nacional es de $90 \%$.

*** $89 \%$ de pacientes no testeados (año 2011).
} 
TB/VIH. Así mismo, se deben establecer estrategias coordinadas con otros sectores que se relacionan con las poblaciones de riesgo para TB: población privada de libertad, personas viviendo en situación de calle, diabéticos, personas con problemas de drogadicción y alcoholismo, problemas de salud mental, migrantes, etc.

Finalmente, se recomienda implementar un plan de fortalecimiento de la red de laboratorios de TB que asegure una infraestructura, equipamiento y recursos humanos que permitan aumentar la actividad de localización de casos, cumpliendo con los estándares de bioseguridad. Este plan debería contemplar la descentralización de la técnica del cultivo a nivel intermedio.

\section{Comentarios}

La deteriorada situación del Programa de Control de la Tuberculosis de Chile observada por los miembros de la misión de OPS no es algo desconocido para los encargados nacionales del PROCET. A lo largo de las múltiples visitas de supervisión y asesoría que se han realizado durante los años 2011 y 2012 a casi todos los Servicios de Salud del país, hemos podido constatar que las deficiencias señaladas en este informe se repiten en cada uno de los Servicios de Salud en mayor o menor medida, lo que ha sido reiteradamente informado a las autoridades ministeriales.

La importante disminución de las tasas de incidencia de tuberculosis en Chile en los años previos a 2007, acompañada de la pérdida de visión de salud pública que rige nuestro sistema de salud en la actualidad, ha quitado prioridad al Programa en todos los niveles, determinando la disminución de los recursos financieros necesarios para el desarrollo de las actividades operacionales, con lo que el diagnóstico y tratamiento exitoso de los casos ha disminuido, deteriorando finalmente la situación epidemiológica y comprometiendo gravemente la posibilidad de la eliminación de la enfermedad como Problema de Salud Pública al año 2020. El mayor temor viene dado por la posibilidad de reversión de la curva de descenso, debido a la acumulación de fuentes de contagio en la población a consecuencia del diagnóstico tardío, situación que ya se ha visto acontecer en otros países donde las medidas de control de la tuberculosis se relajaron al igual que lo que está sucediendo en Chile ${ }^{3}$.

Hacemos nuestras, entonces, las recomendaciones de la OPS, destacando que dentro de la estrategia DOTS de la OMS, el primer punto corresponde al compromiso político de los gobiernos para garantizar una financiación aumentada y sostenida ${ }^{4}$. Es urgente comprometer desde el nivel ministerial a los gestores de la red asistencial para alcanzar las metas del PROCET en la localización de casos y en su tratamiento oportuno y efectivo, lo que incluye asegurar los horarios necesarios a los Equipos de Tuberculosis para desarrollar sus acciones y garantizar una red de laboratorios que pueda cubrir el aumento de la demanda de exámenes de pesquisa que debería producirse.

Finalmente, es necesario agradecer la disposición de los miembros de la Misión de la OPS, ya que su presencia en Chile constituye un apoyo fundamental en nuestra lucha por alcanzar la eliminación de la tuberculosis como Problema de Salud Pública en Chile en los plazos fijados por el Ministerio de Salud.

\section{Bibliografía}

1.- PROGRAMA NACIONAL DE CONTROL Y ELIMINACIÓN DE LA TUBERCULOSIS. Informe de situación 2008-2011. Ministerio de Salud. Santiago. 2012.

2.- OFICINA REGIONAL DE TUBERCULOSIS. Misión de Monitoreo y Evaluación Externa al Programa de Control de la Tuberculosis de Chile. Santiago, 10 de diciembre del 2012.

3.- MARRERO A, CARRERAS L, SANTIN M. El Programa de Control de la Tuberculosis en Cuba. Rev Cubana Med Gen Integr 1996; 12: 381-5.

4.- ORGANIZACIÓN MUNDIAL DE LA SALUD. Tuberculosis: Proseguir la expansión y mejora de un DOTS de calidad. [En línea] consultado: 7 de febrero del 2013 en http://www.who.int/tb/dots/es/.

Correspondencia a:

Dra. Tania Herrera M.

Directora, Programa de Control y Eliminación de la

Tuberculosis- PROCET.

Ministerio de Salud. Santiago. Chile.

E-mail: tania.herrera@minsal.cl 